Ann. Génét. Sél. anim., 1969, 1 (3), 219-226.

\title{
HÉRÉDITÉ DE LA COULEUR BLANCHE DU MOUTON BERRICHON CROISÉ A DES SOLOGNOTS
}

\author{
J.-J. LAUVERGNE \\ Station centrale de Génétique animale, \\ Centre national de Recherches zootechniques, 78 -Jouy-en-Josas \\ Institut national de la Recherche agronomique
}

\section{SOMMAIRE}

La couleur blanche uniforme du Berrichon semble déterminée par un mutant autosomal de panachure blanche dont l'expressivité a été modifiée par sélection jusqu'à extension totale du blanc chez l'homozygote et même chez l'hétérozygote. Par le jeu de l'absence ou de la présence de polygènes le caractère blanc peut apparaftre de type monofactoriel dominant à l'intérieur de la race alors que sa dominance semble intermédiaire voire absente lors de croisements avec des races uniformément colorées. Cette explication rendrait compte d'apparentes anomalies de ségrégation signalées à propos de la couleur blanche dans d'autres races ovines.

\section{INTRODUCTION}

La couleur blanche de la toison des moutons est due à l'absence de pigments mélaniques; elle est très répandue parmi les races améliorées de moutons originaires d'Europe, car elle répond à une exigence de technologie lainière.

Génétiquement, cette couleur n'a pas été obtenue à partir d'un gène d'albinisme car même lorsque le corps, la tête et les membres sont totalement blancs, les yeux et certaines muqueuses restent colorés par un pigment mélanique.

En France, on croise fort couramment les béliers berrichons tout blancs à des brebis de race solognote uniformément colorées $\left({ }^{1}\right)$. Les produits de première génération présentent une panachure variable. Afin de vérifier l'existence d'un facteur mendélien pour la panachure et de préciser son déterminisme héréditaire, on a entrepris, ces dernières années, des croisements de retour sur les deux races parentales.

(1) du moins à la naissance où l'agneau est tout brun; par la suite la toison laineuse devient blanche mais il s'agit d'une canitie précoce ayant une autre origine génétique. 


\section{I. - MATÉRIEL ET MÉTHODES}

\section{A. - Animaux observés}

Les croisements ont été effectués à la ferme expérimentale de La Sapinière à Osmoy près de Bourges (Cher) et dans le troupeau d'un éleveur de Sologne à Bièvre par Marcilly-en-Gault (Loiret-Cher).

Les $F_{1}$ ont été observés à La Sapinière (1965) et à Bièvre (1966), les back-cross avec le Solognot à La Sapinière $(1966,1967,1968$ et 1969) et les back-cross avec le Berrichon à Bièvre (1966). Seul, le premier back-cross a été obtenu à partir d'animaux déjà examinés en $\mathrm{F}_{1}$.

Sur des silhouettes où les animaux sont présentés de profil à droite et à gauche, un même observateur a reporté le dessin des panachures de tous les animaux de l'expérience.

\section{B. - Établissement d'une échelle de panachure}

Plusieurs auteurs ont déjà conçu et utilisé des échelles pour mesurer la panachure du mouton. Parnell (1939), en race Blackface d'Écosse, a imaginé deux systèmes de notation, l'un pour la tête, l'autre pour le corps et les pattes, en 6 et 7 degrés. Les notes sont, en gros, proportionnelles à l'extension de la surface pigmentée. TERriLl (1947) aux États-Unis a utilisé un système semblable pour mesurer l'extension des taches sur les pattes en races Targhee et Columbia. En Turquie, DüGzưNEs et coll. (1960) ont employé trois échelles de notation, l'une pour la tête (de 0 à 4), l'autre pour le corps (de 0 à 5 ) et la troisième pour les pattes $(0$ ou 1$)$. En additionnant les trois notes, ils obtiennent une note globale pour la panachure. Cette mesure est appliquée à l'étude d'un croisement entre les races Mérinos et Akaraman. Pour l'étude de la couleur noire qui apparaît de temps en temps en race Mérinos d'Australie, Kelley et Shaw (1942) se contentent deux degrés : - white "lorsqu'il n'y a pas de grandes taches colorées visibles et "pigmented "lorsque la coloration se présente sous forme d'au moins une tache visible. Brooker et DoLLING (1965) ont quelque peu perfectionné l'échelle en distinguant trois génotypes : white, black spot (lorsqu'il y a une petite tache de laine pigmentée) et black (tous les cas où l'extension est plus importante). Quant à Hayman et Cooper (1964), toujours sur le Mérinos australien, ils utilisent une échelle à 4 degrés : " white", "black spotted " (une seule tache colorée sur le corps ou un groupe de petits points colorés), " piebald "(taches irrégulières sur le corps), "head pattern "(du blanc sur la tête, la queue et le bas des pattes).

Ces échelles ne conviennent pas à notre étude, car elles ne font pas apparaitre nettement un phénomène qui nous a semblé très important : le critère d'ordre dans l'apparition des taches colorées. En effet, lorsque nous classons nos silhouettes, de la plus claire à la plus foncée, même grossièrement, il est facile de distinguer plusieurs stades : tout d'abord les taches se localisent sur les pattes, puis elles s'agrandissent et elles apparaissent sur la tête; au stade suivant, c'est le cou qui est marqué, ensuite, et seulement à ce moment-là, on distingue les premières taches sur le corps, qui se développeront pour donner une panachure plus ou moins variable. taches.

L'échelle que nous avons utilisée tient compte de cette hiérarchie dans la localisation des

Les quatres premiers degrés (tout blanc; pattes colorées; pattes et tête ou cou colorés; pattes, et cou colorés) sont facilement isolables. Lorsqu'il s'agit de classer les phénotypes où le corps est pigmenté, on n'a plus les mêmes facilités car les ultimes taches blanches n'ont pas une localisation aussi précise que les premières taches colorées. On a cependant fait trois classes 4,5 et 6 de plus en plus colorées, l'ultime degré étant atteint en classe 7 où l'animal est uniformément coloré.

$$
\text { C. - Influence du coté }
$$

Sur 123 animaux de première génération, examinés des deux côtés, 21 (17\%) étaient notés différemment à gauche et à droite. Ce chiffre était de deux pour 80 back-cross avec le Berrichon $(2,5 \%)$ et de zéro pour l'autre back-cross.

En aucun cas, la différence n'excédait une unité de l'échelle de panachure adoptée et, une une fois sur deux, elle était en faveur de l'un des deux côtés.

Ainsi, la variation intra-animal est faible et aucun côté ne semble favorisé. Comme nous l'avons fait chez les bovins, (LAUvergne, 1966), il nous suffira d'en considérer un seul; la péréquation s'établira d'elle-même. Nous avons choisi d'examiner le côté droit.

\section{II. - RÉSULTATS}

Les résultats des différents croisements sont donnés sexe par sexe dans le tableau I où l'on a également procédé aux regroupements des sexes et des $F_{1}$. Les histogrammes de la figure 2 sont établis à partir des données regroupées. 


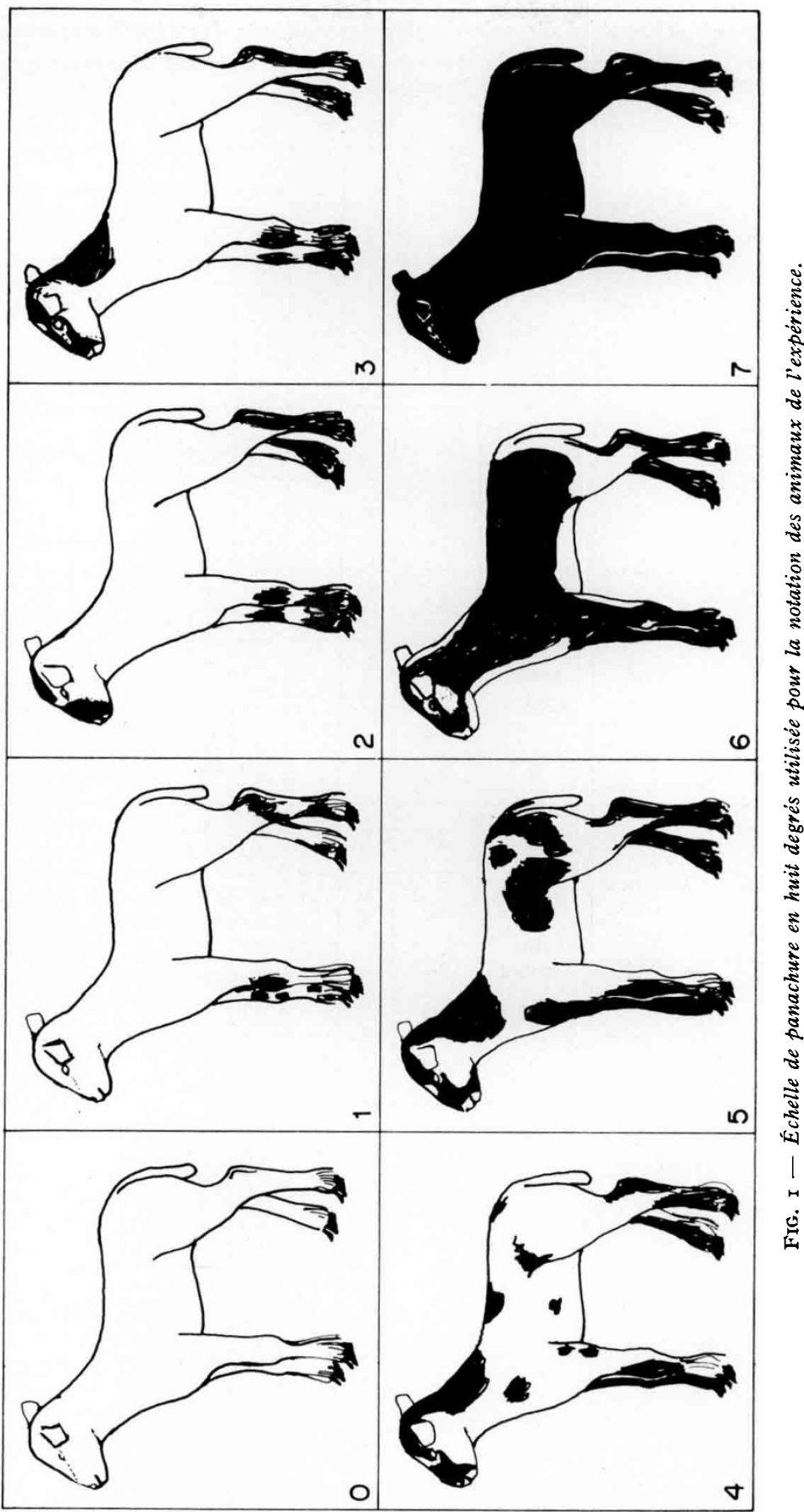


TABLEAU I

Classement, selon l'échelle colorée, des descendants des divers croisements.

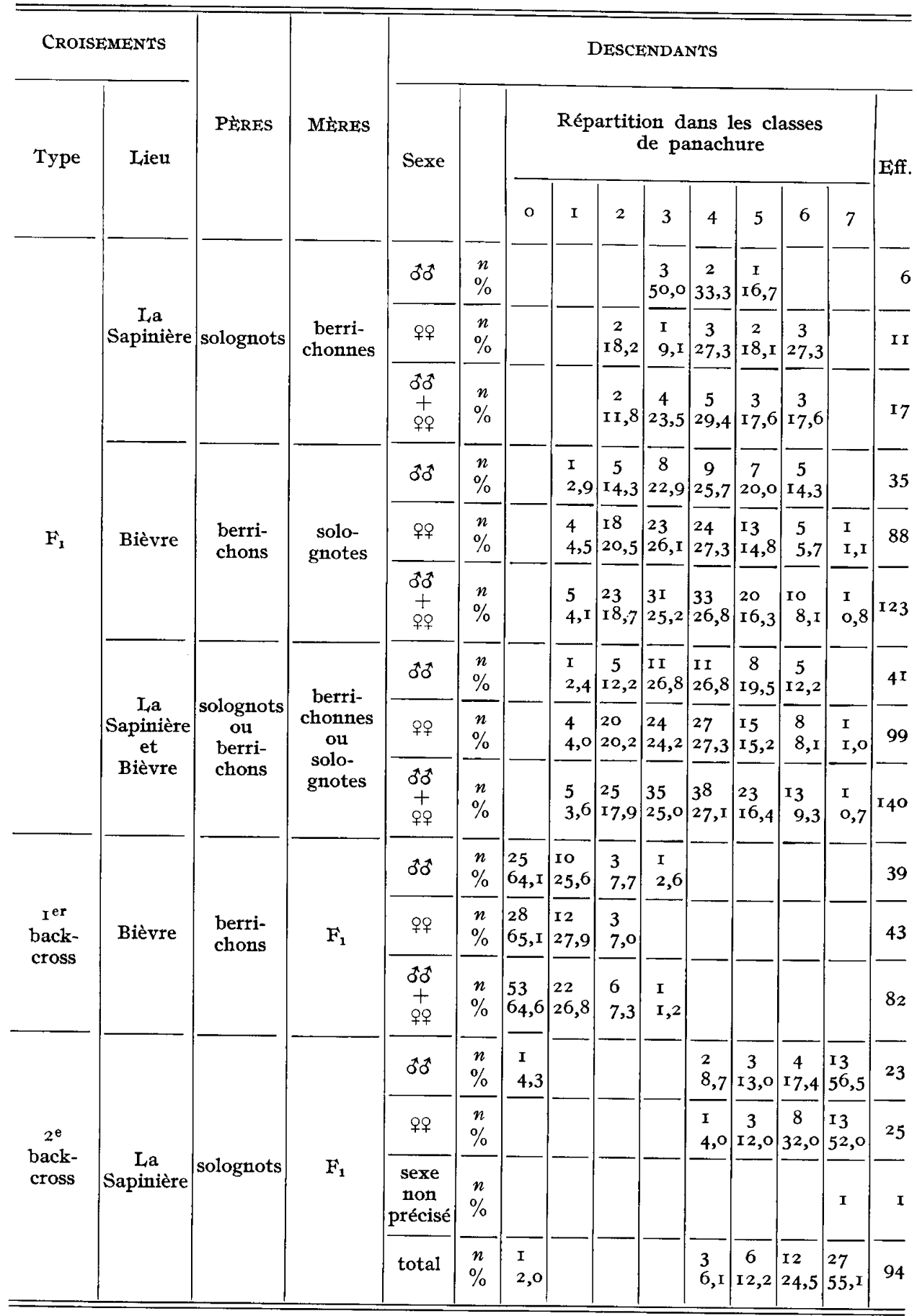




\section{III. - DISCUSSION ET CONCLUSION}

Compte tenu des effectifs, les fréquences à l'intérieur du même type de croisements sont suffisamment rapprochées chez les mâles et chez les femelles pour que l'on puisse admettre que le sexe n'a pas d'influence sur la répartition des taches.
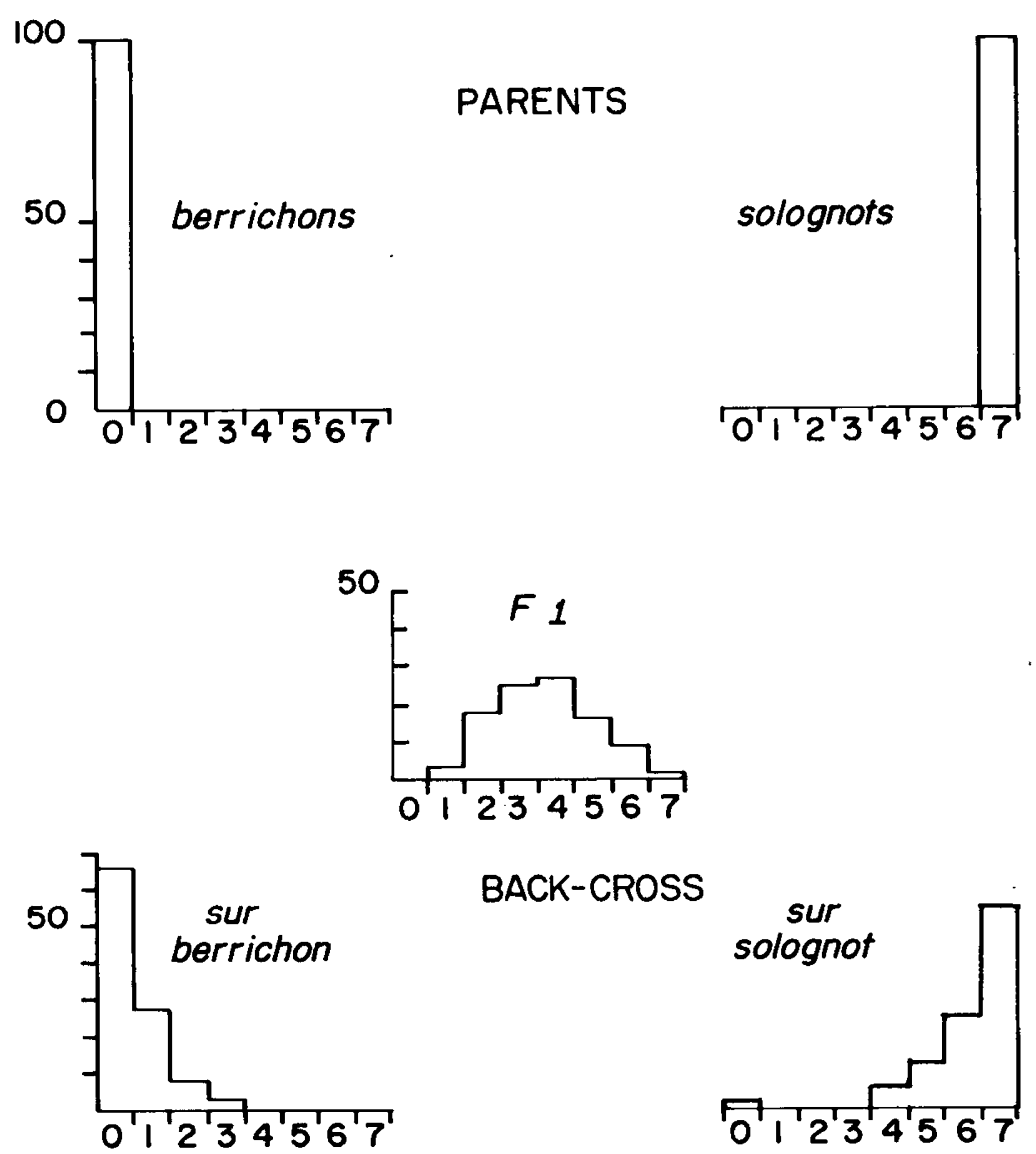

BACK-CROSS

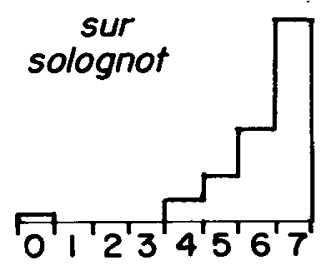

FIG. 2 - Histogrammes montrant la répartition des descendants des divers croisements dans les classes de panachure.

En $F_{1}$ on a pu examiner les produits des deux croisements directs et réciproques; à première vue la répartition des descendants en classes de panachure est la même dans les deux cas.

Ainsi, pour la discussion génétique est-il besoin de ne tenir compte que des données regroupées telles qu'elles sont présentées dans la figure 2 .

En n'observant que la $F_{1}$, on pourrait estimer que 1'extension du blanc est purement polygénique, mais l'examen des 2 back-cross laisse à penser qu'il existe un gène majeur. On retrouve, en effet, des accumulations d'au moins 50 p. Ioo des données dans les classes parentales (fig. 2). Eǹ outre une hypothèse purement 
polygénique est bien moins évidente que celle qui admet que le blanc du Berrichon est dû à un gène muté à dominance intermédiaire dont l'expressivité est soumise à une action polygénique.

Dans cette hypothèse monofactorielle, la pénétrance serait complète chez les hétérozygotes Berrichon $\times$ Solognot de la $\mathrm{F}_{\mathrm{I}}$. La variation de panachure observée serait attribuable aux modificateurs de type polygène provenant plutôt de la race uniformément colorée, car on peut penser que dans la race uniformément blanche, on a obtenu par une longue sélection, l'homozygotie de ces polygènes modificateurs pour l'extension du dessin blanc. Cette explication n'est pas en désaccord avec les résultats des deux back-cross. Dans le back-cross avec la race blanche où l'on retrouve chez tous les animaux un fond de $50 \mathrm{p}$. Ioo de ces modificateurs, les homozygotes n'auront aucune peine à être tout blancs, ainsi qu'un certain nombre d'hétérozygotes comme 1'indique le pourcentage de $64,6 \mathrm{p}$. Ioo d'animaux tout blanes significativement supérieur à 50 p. I00 $\left(\chi^{2}=7,024\right.$ à I d. 1.). Dans le back-cross avec la race colorée, au contraire les deux catégories uniformément colorées et panachées $(27$ et 22 ) se répartissent dans des proportions qui ne s'écartent pas significativement $d u$ rapport $I$ : I $\left(\chi^{2}=0,5 I 0 ;\right.$ I d. 1.). A une exception près, aucun hétérozygote n'est tout blanc, le parent solognot n'apportant pas suffisamment de modificateurs pour que le seuil soit franchi, on a la même situation qu'en $\mathrm{F}_{1}$ où, même avec les 50 p. Ioo de polygènes du Berrichon, aucun sujet n'était tout blanc. Les données de ce second back-cross s'accordent en outre avec celles de la $F_{1}$ pour préciser que la dominance du facteur pour la panachure est totale ou subtotale.

S'il reste quelque doute en faveur d'une hypothèse purement quantitative, il serait intéressant pour les dissiper d.e procéder à des croisements $F_{1}$ par $F_{1}$.

La conception à laquelle nous parvenons quant au déterminisme de la couleur blanche du Mouton berrichon a des implications qui dépassent le cadre de la race. On peut remarquer en effet que les derniers stades de la disparition des taches colorées dans nos croisements correspondaient tout à fait au standard de certaines races à toison blanche et à extrémités colorées telles que RoBERTS (I928) par exemple les décrit en Angleterre ou telles que l'on peut encore trouver en France (QUITTET, I965) et dans certains pays d'Europe continentale. De là à penser que plusieurs races blanches et des races à tête et pattes colorées d'Europe occidentale tiennent leur coloration du même facteur de panachure dont la sélection a été poussée plus ou moins loin, il n'y a qu'un pas assez facile à franchir si l'on se rappelle que toutes ces races ont des origines communes.

On pourrait aussi expliquer un certain nombre d'observations parfois contradictoires en se rappelant le rôle joué par la sélection polygénique modifiant 1'expressivité de la panachure.

Tout d'abord, on comprendrait pourquoi la couleur noire ou brune uniforme, qui apparaît dans certains troupeaux blancs, est, à première vue, déterminée par un facteur récessif (troupeau de Graham Bell, DAvENPORT,I905; Bleu du Maine de M. Chollet-Molines, LAuvergne, I96I) : en présence des modificateurs, 1'hétérozygote serait généralement incapable de présenter une quelconque plage colorée. Les exceptions continuellement rencontrées depuis DAVENPORT (rgo5) par de nombreux auteurs dont nous citerons : DRY (I924) sur le Wensleydale, HaymaN 
et COOPER (1964) sur le Mérinos d'Australie, seraient dues à un manque accidentel de modificateurs chez des hétérozygotes.

En deuxième lieu, on s'expliquerait mieux pourquoi la couleur blanche d'une race qui se comporte d'une manière dominante vis-à-vis des quelques agneaux noirs qui apparaissent en son sein est, au contraire, récessive lors de croisement avec certaines races colorées étrangères. Ces races étrangères non seulement ne porteraient aucun gène modificateur favorable à l'extension des taches provoquées par la présence d'un gène de panachure mais au contraire des limitateurs d'extension du dessin blanc acquis par sélection contre l'expressivité d'un éventuel autre facteur de panachure tel que l'étoile en tête. Ceci serait le cas du Mérinos dont la couleur blanche se montre récessive lors $\mathrm{du}$ croisement avec des races colorées d'origine asiatique (TäNZER, I932).

Sans insister plus longtemps dans le cadre de cet article, nous pouvons conclure en disant que le résultat de nos expériences de croisement font entrevoir une explication qui peut sans doute s'appliquer à plusieurs races blanches d'Europe occidentale ayant des origines communes : leur couleur proviendrait d'un mutant de panachure dont l'expressivité, la pénétrance et, à la limite, la dominance ont évolué par sélection en faveur de polygènes modificateurs du dessin blanc.

Rę̧u pour publication en novembre 1969.

\section{REMERCIEMENTS}

Nous tenons à remercier Monsieur Jacques de la Selle qui a mis une partie de son troupeau à notre disposition pour réaliser le $1^{\mathrm{er}}$ back-cross, ainsi que Monsieur SımoN, berger à Bièvre par Marcilly-en-Gault (Loir-et-Cher).

\section{SUMMARY}

HEREDITY OF WHITE COLOUR OF BERRICHON SHEEP CROSSED TO SOLOGNOT

First generation crossed and both back crosses were performed between white Berrichon sheep and solid-coloured Solognot. In $140 \mathrm{~F}_{1}$, all animals are piebald; $64 \%$ cf 82 back-crosses on Berrichon are white and $36 \%$ piebald; $55 \%$ of 45 back-crosses with Solognot are solid-coloured and $45 \%$ piebald or white.

The simplest genetic interpretation is that the white colour of Berrichon was determined by an autosomic piebald factor, the expressivity of which depends on a quantitative polygenic selection. Because of the very numerous homozygous polygens in the white breed, the white factor was dominant inside the race. In $F_{1}$ and back-crosses the relative loss of polygens was responsible for the observed variations in piebaldenss. The numerous reversals of dominance occuring in various white sheep breeds could be explained in the same way. In many cases these breeds have some common ancestor with Berrichon sheep.

\section{RÉFÉRENCES BIBLIOGRAPHIQUES}

Brooker M. G., Dolling C. H. S., I965. Pigmentation of sheep. I. Inheritance of pigmented wool in the Merino. Austr. J. Agric. Res., 16, 219-228.

DAVENPORT C. B., I905. The origin of black sheep in the flock. Sciences (N. S.), 22, 674.

DüzgüNes O. I. YARKIN I., SöNMEZ R., I960. A study on the variation in colour in the fat tailed white Karaman sheep, Merino-fleisch Shafe and their crosses. Z. Tierzücht. ZüchtBiol., 74, 36-47. 
HaYman R. H., COOPER D. W., r964. The inheritance of black pigmentation in a family of inbred Peppin Merinos. Austr. J. Agric. Res., 15, 453-460.

Kelley R. B., Shaw H. E. B., x942. Note on the occurence and inheritance of pigmented wool. $J$. Coun. Sci. Industr. Res. A ust., 15, I-3.

LAUVERGNE J.-J., I96r. Sur le déterminisme génétique de la couleur noire dans la race « Bleu du Maine ». Ann. Génét., 2, 47:52.

I.AUVERGNe J.-J. 1966. Contribution à l'étude de l'hérédité de la couleur du pelage chez les bovins domestiques, 57 p. These $3^{\mathrm{e}}$ cycle Fac. Sci. Paris.

Parnell J. W., 1939. Notes on the history of the scottish mountain blackface sheep and some genetical observations on certain breed characteristics. Scient. A gric., 20, 205-233.

QUITTET E., r965. Races ovines françaises, 96 p. La maison Rustique, Paris.

Roberxs J. A. F., r928. Colour inheritance in sheep. III. Face and leg colour. J. Genet., 19, $261-268$.

TÄNzer E., I932. Neuere Untersuchungen über die Vererbung beim Karakulschaf. Z. Indukt. Abstamm.-u. VererbLehre, 61, 62-75.

TERRII C. E., 1947. Color on the legs of sheep. J. Hered., 38, 89-92. 